\title{
NILPOTENT ORBITS OF EXCEPTIONAL LIE ALGEBRAS OVER ALGEBRAICALLY CLOSED FIELDS OF BAD CHARACTERISTIC
}

\author{
D. F. HOLT and N. SPALTENSTEIN
}

(Received 5 May 1983)

Communicated by R. Lidl

\begin{abstract}
The classification of the nilpotent orbits in the Lie algebra of a reductive algebraic group (over an algebraically closed field) is given in all the cases where it was not previously known $\left(\mathbf{E}_{7}\right.$ and $\mathbf{E}_{8}$ in bad characteristic, $\mathbf{F}_{4}$ in characteristic 3 ). The paper exploits the tight relation with the corresponding situation over a finite field. A computer is used to study this case for suitable choices of the finite field.
\end{abstract}

1980 Mathematics subject classification (Amer. Math. Soc.): 20 G 99, 17 B 25, 17 B 45

Let $g$ be the Lie algebra of a connected reductive algebraic group $G$ defined over an algebraically closed field $k$ of characteristic $p$ ( 0 or a prime). The classification of nilpotent orbits in $g$ reduces easily to the case where $G$ is simple, and it is known in most cases. If $p$ is good for $G$ (that is, not bad [20, page 178]), Springer's correspondence [20, page 229] allows us to use the classification of unipotent elements in $G$ ([20], [2], [7], [13], [11], [12]). If $p$ is bad, the following cases are known: $G$ classical [5], $G_{2}$ [23], $E_{6}[17], F_{4}$ when $p=2$ [18].

The remaining cases are
(a) $F_{4}, p=3$,
(b) $E_{7}, p=2,3$,
(c) $E_{8}, p=2,3,5$.

They are dealt with in this paper.

If $k$ is an algebraic closure of a finite field $\mathbf{F}_{q}$ and $G$ is defined over $\mathbf{F}_{q}$, it should be possible to use a computer in the study of the action of $G^{F}$ on $\mathfrak{g}^{F}$, where $F$

(c) 1985 Australian Mathematical Society $0263-6115 / 85 \$ A 2.00+0.00$ 
denotes the Frobenius morphisms of $G$ and $g$, and this would yield much information on the action of $G$ on $\mathfrak{g}$. Let $U$ be an $F$-stable maximal unipotent subgroup of $G$, with Lie algebra $u$. Instead of looking directly at the action of $G^{F}$ on $g^{F}$, the computer is used in this paper to calculate the order of the stabilizer in $U^{F}$ of various elements of $\mathfrak{u}^{F}$. As far as programming is concerned, this has the advantage that there are efficient algorithms for finite $p$-groups.

As a corollary of the classification, we get

THEOREM 1. There are only finitely many nilpotent orbits in $\mathfrak{g}$.

It would of course be desirable to have a unified proof, as for unipotent classes [8].

For $x \in \mathfrak{g}$, let $G_{x}$ denote the stabilizer of $x$ in $G$ and let $\mathfrak{B}_{x}^{G}$ be the variety of all Borel subgroups of $G$ whose Lie algebra contains $x$.

THEOREM 2. $\operatorname{dim} G_{x}=2 \operatorname{dim} \mathfrak{B}_{x}^{G}+r$, where $r$ is the rank of $G$.

We may assume that $G$ is of type $F_{4}$, with $p=3$, or of type $E_{7}$ or $E_{8}$, with $p$ bad. We say that $x \in \mathrm{g}$ is distinguished if it is nilpotent and $Z^{0}(G)$ is a maximal torus of $G_{x}$ (this differs slightly from the definition given in [2]). As noticed in [15], it is sufficient to prove that the distinguished orbits can be obtained by the process of induction [10], and this is actually how we shall get hold of them.

The theory of Springer representations can be made to work in bad characteristic ([3], [9]), and Theorem 2 implies that the nilpotent orbits can be parametrized by irreducible representations of the Weyl group.

The method used here does not give the structure of $G_{x}(x \in \mathfrak{g}$ nilpotent), nor the inclusion relation between closures of nilpotent orbits. The results are therefore weaker than those obtained for unipotent elements by Mizuno [12].

The proof proceeds along the following lines. Let $F_{q}$ be a finite field of characteristic $p$. We can assume that $k$ is an algebraic closure of $\mathbf{F}_{q}$ (this is used by Lusztig [8] in his proof of the finiteness of the number of unipotent classes) and that $G$ is defined and split over $\mathbf{F}_{q}$. Let $F: \mathfrak{g} \rightarrow \mathfrak{g}$ be the Frobenius morphism of g. Let $C$ be an $F$-stable nilpotent orbit in $g$, let $x \in C$ and let $S$ be a maximal torus of $G_{x}$. Then $\left|C^{F}\right|$ is given by a polynomial in $q$ which depends only on $\operatorname{dim} C$ and the conjugacy class of $S$ in $G$. The computer is used for the following two major steps:

(i) Let $C_{1}, \ldots, C_{m}$ be the non-distinguished nilpotent orbits in $\mathrm{g}$. We compute $\operatorname{dim} C_{i}$, and hence $\left|C_{i}^{F}\right|$, for $1 \leqslant i \leqslant m$.

(ii) We construct distinguished orbits $C_{m+1}, \ldots, C_{n}$ such that $\sum_{1 \leqslant i \leqslant n}\left|C_{i}^{F}\right|=q^{2 N}$ (as polynomials), where $N$ is the number of positive roots of $G$. As the number of 
nilpotent elements in $\mathrm{g}^{F}$ is $q^{2 N}[19]$, this shows that $C_{1}, \ldots, C_{n}$ are all the nilpotent orbits in $\mathrm{g}$.

The second named author is grateful to the University of Warwick for its hospitality, and to the Science and Engineering Research Council for financial support during the preparation of this paper.

\section{Notation}

In addition to that used in the introduction, we shall use the following notation.

If $H$ is an affine algebraic group, we write $U_{H}$ or $R_{u}(H)$ for its unipotent radical.

We fix a Borel subgroup $B$ of $G$, with maximal unipotent subgroup $U$, and a maximal torus $T$ in $B$. The Weyl group of $G$ is $W=N_{G}(T) / T$. More generally, if $H \supset T$ is a connected subgroup of $G$, let $W_{H}=N_{H}(T) / T \subset W$. Let $w_{H}$ be the element of maximal length in $W_{H}$ (i.e. $w_{H}$ is the unique element of $W_{H}$ such that $H \cap B \cap{ }^{w_{H} B} \subset T U_{H}$ ).

For the Lie algebra of an algebraic group we use the corresponding gothic letter, for example $\mathfrak{b}, \mathfrak{u}, \mathfrak{u}_{H}$ are the Lie algebras of $B, U, U_{H}$ respectively. We write $g \cdot x$ for $\operatorname{Ad}(g)(x)(g \in G, x \in g)$. The nilpotent variety of $g$ is denoted $\mathscr{N}$.

Let $\Phi \subset \operatorname{Hom}\left(T, \mathbf{G}_{m}\right)$ be the root system of $G$, with $\Phi^{+}$the set of positive roots and $\Delta$ the basis corresponding to $B$. For each $\lambda \in \Phi$, let $x_{\lambda}: \mathbf{G}_{a} \rightarrow G$ be adapted to $\lambda$. Let $U_{\lambda}=x_{\lambda}\left(G_{a}\right) \subset G$ and $X_{\lambda}=\left(d x_{\lambda}\right)_{0}(1) \in \mathfrak{g}$. Let also $L_{\lambda}$ be the subgroup of $G$ generated by $U_{\lambda}$ and $U_{-\lambda}$. The roots are denoted as in [4]. For example, for $F_{4}$ the highest root is 2342 , and for $E_{8}$ it is ${ }_{3}^{2465432}$.

Except in paragraph $1, k$ is an algebraic closure of a finite field $F_{q}$. We assume that $G, B, T$ and $x_{\lambda}(\lambda \in \Phi)$ are defined over $F_{q}$, and $F$ denotes the Frobenius morphisms of $G, g$, etc. The conventions concerning Lang's theorem and its applications are the same as in [20]. For example, if $T^{\prime}$ is an $F$-stable maximal torus of $G$ corresponding to $w \in W$, then $F$ acts on $T^{\prime}$ as $w F$ on $T$.

The classification of nilpotent orbits for $E_{7}$ and $E_{8}$ in bad characteristic was conjectured in [16], with a notation not adopted in this paper since it conflicts with the Bala-Carter method which we shall use constantly.

There are several references to results concerning unipotent classes in $G$ instead of nilpotent orbits in $\mathrm{g}$. In most cases the demonstrations can be transposed easily, but some care is needed in the use of the process of induction [10]. This is discussed in paragraph 5 .

\section{Reduction to $\overline{\mathbf{F}_{p}}$}

For completeness we show why it is enough to consider the case of an algebraic closure of $\mathbf{F}_{p}$. Let $k_{0}$ be the algebraic closure of the prime field in $k$. Then $G$ can 
be obtained by extension of scalars from a reductive algebraic group $G_{0}$ defined over $k_{0}$. The nilpotent variety $\mathscr{N}$ is likewise obtained by extension of scalars from the nilpotent variety $\mathscr{N}_{0}$ of $\mathfrak{g}_{0}$, as well as the adjoint action. Suppose that the number of $G_{0}$-orbits in $\mathrm{g}_{0}$ is finite. Let $C_{1}, \ldots, C_{n}$ be these orbits and let $x_{i} \in C_{i}$ $(1 \leqslant i \leqslant n)$. Let $X_{0}$ be the disjoint union of $n$ copies of $G_{0}$ and let $f_{0}: X_{0} \rightarrow \mathscr{N}_{0}$ be the morphism which sends the element $g$ of the $i$ th copy to $g \cdot x_{i}$. Then $f_{0}$ is surjective. It is a general fact that if $\pi_{0}: Y_{0} \rightarrow Z_{0}$ is a surjective morphism of $k_{0}$-varieties, then the morphism $\pi: Y \rightarrow Z$ obtained by extension of scalars to $k$ is also surjective. Therefore the morphism $f: X \rightarrow \mathscr{N}$ from the disjoint union of $n$ copies of $G$ to $\mathscr{N}$, defined by $g \mapsto g \cdot x_{i}$ on the $i^{\text {th }}$ copy, is also surjective. There are thus at most $n$ nilpotent orbits in $\mathrm{g}$. On the other hand, the closure $\bar{C}_{i}$ of $C_{i}$ in $\mathrm{g}$ is $G_{0}$-stable, hence $G$-stable since $G_{0}$ is dense in $G$, and if $x_{j} \in \bar{C}_{i}$ then $x_{j}$ is also in the closure of $C_{i}$ in $\mathfrak{g}_{0}$. This implies that $x_{1}, \ldots, x_{n}$ form a system of representatives for the $G$-orbits in $g$.

\section{The polynomial $\left|C^{F}\right|$}

Let $C \subset \mathrm{g}$ be an $F$-stable nilpotent orbit. We want to show that $\left|C^{F}\right|$ is given by a polynomial in $q$ of very special form.

Let $x \in C$, let $S$ be a maximal torus of $G_{x}$ and let $M=C_{G}(S)$. Then $M$ is a Levi factor of some parabolic subgroup $P$ of $G, S=Z^{0}(M)$ and $x$ is distinguished in $\mathrm{m}$ [2]. The finite group $W_{M, G}=N_{G}(M) / M \cong N_{W}\left(W_{M}\right) / W_{M}$ acts on $M$ and on $\mathrm{m}$.

LEMMA 1. $W_{M, G}$ acts trivially on the set of distinguished orbits in $\mathrm{m}$.

We can assume that $G$ is simple. Then $M$ has at most one factor which is not of type $A$. For type $A$ the only distinguished orbit is the regular nilpotent orbit. If there is a factor of type other than $A$ and $W_{M, G}$ acts by outer automorphisms on it, then it is of type $D_{n}(n \geqslant 4)$ or $E_{6}$. In both cases the nilpotent orbits are known, and the distinguished ones are stable under outer automorphisms.

LEMMA 2. In the situation above, it is possible to choose $x$ and $S$ both F-stable in such a way that $P$ is also F-stable.

We certainly can arrange to have $S$ contained in $T$. Then $S$ and $P$ are $F$-stable. Moreover Lemma 1 shows that $C \cap \mathrm{m}$ is a single distinguished orbit in $\mathrm{m}$. As it is $F$-stable we can find $x \in(C \cap \mathrm{m})^{F}$. 
Let $H$ be a connected algebraic group defined over $\mathbf{F}_{q}$, let $T_{0}$ be an $F$-stable maximal torus of $H$ and let $W_{H}=N_{H}\left(T_{0}\right) / C_{H}\left(T_{0}\right)$ be the Weyl group of $H$. The following formula is due to Steinberg [22]:

$$
\frac{1}{\left|H^{F}\right|}=\frac{1}{\left|W_{H}\right|} q^{\operatorname{dim} T_{0}-\operatorname{dim} H} \sum_{\omega \in W_{H}} \frac{1}{\left|T_{0}{ }^{w F}\right|} .
$$

Proposition 1. Let $x, S$ be as in Lemma 2 and let

$$
c_{M, G}=\frac{1}{\left|W_{M, G}\right|} \sum_{w \in W_{M, G}} \frac{q^{\mathrm{dim} S}}{\left|S^{\omega F}\right|} .
$$

Then

$$
\left|C^{F}\right|=c_{M, G}\left|\dot{G}^{F}\right| q^{-\operatorname{dim} G_{x}} .
$$

Let $A=G_{x} / G_{x}^{0}$. For each $a \in A$, let $x_{a} \in C^{F}$ correspond to the $F$-conjugacy class of $a$ in $A$. It is easily seen that

$$
\left|C^{F}\right|=\frac{1}{|A|} \sum_{a \in A} \frac{\left|G^{F}\right|}{\left|\left(G_{x_{a}}^{0}\right)^{F}\right|} .
$$

Now $a \in A$ is a coset of the form $g G_{x}^{0}$, with $g \in G_{x}$. As all maximal tori of $G_{x}$ are $G_{x}^{0}$-conjugate, we can assume that $g$ normalizes $S$. Let $W_{x}=N_{G_{x}^{0}}(S) / C_{G_{x}^{0}}(S)$ be the Weyl group of $G_{x}^{0}$ and let $W_{x}^{\prime}=N_{G_{x}}(S) / C_{G_{x}^{0}}(S)$. Then by Steinberg's formula,

$$
\frac{1}{\left|\left(G_{x_{a}}^{0}\right)^{F}\right|}=\frac{1}{\left|W_{x}\right|} q^{\operatorname{dim} S-\operatorname{dim} G_{x}} \sum_{w \in W_{x}} \frac{1}{\left|S^{w g F}\right|},
$$

and therefore

$$
\left|C^{F}\right|=\frac{\left|G^{F}\right|}{\left|W_{x}^{\prime}\right|} q^{\operatorname{dim} S-\operatorname{dim} G_{x}} \sum_{w \in W_{x}^{\prime}} \frac{1}{\left|S^{w F}\right|} .
$$

In order to prove the proposition, it remains only to check that

$$
\frac{1}{\left|W_{x}^{\prime}\right|} \sum_{w \in W_{x}^{\prime}} \frac{1}{\left|S^{w F}\right|}=\frac{1}{\left|W_{M, G}\right|} \sum_{w \in W_{M, G}} \frac{1}{\left|S^{w F}\right|},
$$

and this holds since Lemma 1 implies that the natural homomorphism $W_{x}^{\prime} \rightarrow W_{M, G}$ is surjective.

ReMark. Consider $c_{M, G}$ as a rational function of $q$ and $c_{M, G}\left|G^{F}\right|$ as a polynomial in $q\left(q=p^{e}, e \in \mathbf{N}^{*}\right)$. Then they don't depend on the characteristic. For $E_{7}, E_{8}$ and $F_{4}$ they are therefore essentially contained in [12], [13]. 


\section{Non-distinguished nilpotent orbits}

Let $x \in \mathrm{g}$ be nilpotent and let $C=G \cdot x$. Then all irreducible components of $C \cap \mathfrak{u}$ have the same dimension [14]. For $w \in W$, let $\mathfrak{u}_{w}=\mathfrak{u} \cap{ }^{w} \mathfrak{u}$. The following properties are equivalent ([15], see also [21], [14]):

(1) $\operatorname{dim} G_{x}=2 \operatorname{dim} \mathfrak{B}_{x}^{G}+\operatorname{dim} T$;

(2) $\operatorname{dim} C=2 \operatorname{dim}(C \cap u)$;

(3) $\operatorname{dim} B_{x}^{G}=\operatorname{codim}_{u}(C \cap u)$;

(4) $C$ contains a dense open subset of $\mathfrak{u}_{w}$ for some $w \in W$.

Moreover, if $w$ is as in (4), then $\overline{B \cdot \mathfrak{u}_{w}}$ is an irreducible component of $\overline{C \cap \mathfrak{u}}$, all irreducible components of $\overline{C \cap \mathfrak{u}}$ are of this form, and they all have the same dimension.

Let $P \supset B$ be a parabolic subgroup of $G$, let $M \supset T$ be a Levi factor of $P$ and let $U^{\prime}=U \cap M$. Suppose that $x \in \mathfrak{m}$, and that $M \cdot x$ contains a dense open subset of $\mathfrak{u}^{\prime} \cap{ }^{w} \mathfrak{u}^{\prime}$ for some $w \in W_{M}$. As $\mathfrak{u}^{\prime} \cap{ }^{w} \mathfrak{u}^{\prime}=\mathfrak{u}_{w_{G} w_{M} w}, B \cdot\left(\mathfrak{u}^{\prime} \cap{ }^{w} \mathfrak{u}^{\prime}\right)$ is an irreducible component of $\overline{C \cap u}$. Therefore $\operatorname{dim} C=2 \operatorname{dim}(C \cap u)$ $=2 \operatorname{dim} B \cdot\left(u^{\prime} \cap{ }^{w} u^{\prime}\right)$. But $B$ is the semidirect product of $B \cap M$ and $U_{P}$. For any $y \in M \cdot x \cap \mathfrak{u}^{\prime}$ we have $B_{y}=(B \cap M)_{y}\left(U_{P}\right)_{y}$ and $\operatorname{dim}\left(U_{P}\right)_{y}=\operatorname{dim}\left(U_{P}\right)_{x}$. Moreover, two elements $y, y^{\prime}$ of $M \cdot x \cap \mathfrak{u}^{\prime}$ are in the same $B$-orbit if and only if they are in the same $(B \cap M)$-orbit. It follows that

$$
\operatorname{dim} B \cdot\left(\mathfrak{u}^{\prime} \cap{ }^{w} \mathfrak{u}^{\prime}\right)=\operatorname{dim}(B \cap M) \cdot\left(\mathfrak{u}^{\prime} \cap{ }^{w} \mathfrak{u}^{\prime}\right)+\operatorname{dim} U_{P} \cdot x .
$$

As $\operatorname{dim} M \cdot x=2 \operatorname{dim}(B \cap M) \cdot\left(\mathfrak{u}^{\prime} \cap{ }^{w} \mathfrak{u}^{\prime}\right)$, we get finally.

Proposition 2. Suppose that $x \in \mathfrak{m}$ is nilpotent and that $\operatorname{dim} M_{x}=2 \operatorname{dim} \mathfrak{B}_{x}^{M}$ $+\operatorname{dim} T$. Then: $\operatorname{dim} G \cdot x=\operatorname{dim} M \cdot x+2 \operatorname{dim} U_{P} \cdot x, \operatorname{dim} G_{x}=\operatorname{dim} M_{x}+$ $2 \operatorname{dim}\left(U_{P}\right)_{x}$.

Assuming $\operatorname{dim} M_{x}$ known, we need therefore only to compute $\operatorname{dim}\left(U_{P}\right)_{x}$ to get $\left|C^{F}\right|$. For this we use the following result.

Proposition 3. For any $x \in \mathfrak{m},\left(U_{P}\right)_{x}$ is connected.

We may assume that $x \in \mathfrak{m} \cap \mathfrak{b}$ and that $T$ contains a maximal torus $S$ of $M_{x}$. Since $M \supset C_{G}(S)$, it is clear that $S$ is also a maximal torus of $G_{x}$ and $B_{x}$. Any irreducible component of $B_{x}$ contains therefore an element which normalizes $S$, hence centralizes $S$ since $B$ is solvable and connected. Hence every irreducible 
component of $B_{x}$ meets $C_{G}(S) \subset M$. But the semidirect decomposition $B=(B \cap M) U_{P}$ gives a similar decomposition $B_{x}=(B \cap M)_{x}\left(U_{P}\right)_{x}$. As every irreducible component of $B_{x}$ meets $M,\left(U_{P}\right)_{x}$ must be connected.

COROLlary. Let $x \in \mathrm{m}^{F}$ and let $\left|\left(U_{P}\right)_{x}^{F}\right|=q^{d}$. Then $\operatorname{dim}\left(U_{P}\right)_{x}=d$.

APplication. Using the Appendix with $q=p$, we can compute $\operatorname{dim}\left(U_{P}\right)_{x}$, and hence $\operatorname{dim} G_{x}$ and $\left|(G \cdot x)^{F}\right|$ for $x$ nilpotent non-distinguished in $\mathfrak{g}$, assuming of course that the nilpotent orbits are known for Levi factors of proper parabolic subgroups of $G$. For this paper, this has been done with the help of a computer for $E_{7}$ and $E_{8}$. What was actually computed was $\left|U_{x}^{F}\right|$ and $\left|(U \cap M)_{x}^{F}\right|$. The semidirect decomposition with the help of a computer $U=(U \cap M) U_{P}$ gives then $\left|\left(U_{p}\right)_{x}^{F}\right|=\left|U_{x}^{F}\right| /\left|(U \cap M)_{x}^{F}\right|$.

\section{The case of $F_{4}$}

If $G$ is of type $F_{4}$ and $p=3$, there are 12 non-distinguished nilpotent orbits, as in characteristic 0 . Let $C$ be one of them. With the notation of the previous paragraph, we can find $P(\neq G), M$ and $x \in \mathfrak{m} \cap C$ in such a way that $\operatorname{dim}\left(U_{P}\right)_{x}$ can be computed very easily (by hand), with the following exception for which some care is needed. For the class $\tilde{A}_{2}+A_{1}$ ( $\tilde{A}_{2}$ short, $A_{1}$ long), the computation requires an explicit knowledge of the constants in the commutation formulae. We can use those listed in [13]. In all cases we find that the non-distinguished orbits have the same dimension as the corresponding orbits in characteristic 0.

In particular, with the classes we have so far it is not possible to have $\operatorname{dim} \mathfrak{B}_{x}^{G}=0,1,2$ or 4 . But in this case we know that the number of nilpotent orbits in $\mathfrak{g}$ is finite [17]. Richardson orbits are therefore defined. Starting with a parabolic subgroup having a Levi factor of type $\varnothing, \tilde{A_{1}}, \tilde{A_{1}}+A_{1}$ or $\tilde{A}_{2}+A_{1}$, we get a nilpotent orbit $C_{P}$ such that $\operatorname{dim} \mathfrak{B}_{x}^{G}=0,1,2,4$ respectively for $x \in C_{P}$. This gives 4 distinguished nilpotent orbits. Comparing with the case of large characteristic, we find that we have already $q^{48} F$-stable nilpotent elements. We have therefore all the nilpotent orbits in $g$. The distinguished orbits being Richardson orbits, we see also that theorem 2 holds for $G$.

\section{Induction for nilpotent orbits}

Induction for nilpotent orbits is the following operation. Let $P$ be a parabolic subgroup of $G$ and let $M$ be a Levi factor of $P$. Let $x \in \mathrm{m}$ be nilpotent and let $C=M \cdot x$. Assume that $\operatorname{dim} M_{x}=2 \operatorname{dim} B_{x}^{M}+\operatorname{dim} T$. The induced orbit in $\mathfrak{g}$ is 
the nilpotent orbit $\tilde{C}$ which contains a dense open subset of $C+\mathfrak{H}_{P}$. If $x=0, \tilde{C}$ is the Richardson orbit defined by $P$. In [10] the corresponding situation for unipotent elements is investigated, and the finiteness of the number of unipotent classes implies the existence of the induced class. We cannot use this argument here, but we still have:

(1) If $\tilde{C}$ exists, then all the properties described in [10] hold. In particular, if $\tilde{x} \in\left(x+\mathfrak{u}_{P}\right) \cap \tilde{C}$, then $P_{\tilde{x}} \subset M_{x} U_{P}, G_{\tilde{x}}^{0}=P_{\tilde{x}}^{0}$ and $\operatorname{dim} G_{\bar{x}}=\operatorname{dim} M_{x}=2 \operatorname{dim} \mathfrak{B}_{\tilde{x}}^{G}$ $+\operatorname{dim} T$.

(2) If there exists $\tilde{x} \in x+\mathfrak{u}_{P}$ such that $\operatorname{dim} P_{\bar{x}} \leqslant \operatorname{dim} M_{x}$, then the induced orbit $\tilde{C}$ exists and $\tilde{x} \in \tilde{C}$.

If $G$ is an exceptional group, induction can be computed explicitely as follows. We assume that Levi factors of proper parabolic subgroups of $G$ have only finitely many nilpotent orbits, that these orbits are known, and that induction inside their Lie algebras is known.

Let $S$ be a maximal torus of $M_{x}$ and let $L=C_{G}(S)$. If $\tilde{C}$ exists, there is an element $\tilde{x} \in\left(x+\mathfrak{u}_{P}\right) \cap \tilde{C}$ such that $S$ contains a maximal torus $\tilde{S}$ of $G_{\tilde{x}}$. Let $\tilde{G}=C_{G}(\tilde{S}), \tilde{P}=P \cap \tilde{G}, \tilde{M}=M \cap \tilde{G}$. Then $\tilde{P}$ is a parabolic subgroup of $\tilde{G}, \tilde{M}$ is a Levi factor of $\tilde{P}$ and the unipotent radical $U_{\tilde{P}}$ of $\tilde{P}$ is $U_{P} \cap \tilde{G}$. Now $\tilde{x} \in(x+$ $\left.\mathfrak{u}_{P}\right) \cap \tilde{\mathfrak{g}}=x+\mathfrak{u}_{\tilde{P}}$. Therefore $\tilde{C}$ contains a dense open subset of $x+\mathfrak{u}_{\tilde{P}}$. As $\tilde{C} \cap \tilde{\mathrm{g}}$ is a single $\tilde{G}$-orbit, $\tilde{G} \cdot \tilde{x}$ must be the orbit of $\tilde{\mathrm{g}}$ induced from $\tilde{M} \cdot x \subset \tilde{\mathrm{m}}$.

There are only finitely many subgroups $H$ of $G$ which contain $L$ and are Levi factors of proper parabolic subgroups of $G$. For each of them, let $\tilde{x}_{H}$ be an element in the orbit in $\mathfrak{h}$ induced from the orbit $(M \cap H) \cdot x \subset \mathfrak{m} \cap \mathfrak{h}$. If $\operatorname{dim} G_{\bar{x}_{H}}=\operatorname{dim} M_{x}$ for some $H$, then $\tilde{C}=G \cdot \tilde{x}_{H}$. If no such $H$ exists, the induced orbit is distinguished or does not exist.

We shall use this information to find the distinguished orbits in $\mathrm{g}$. There will only be a finite number of them, and induced orbits will therefore exist. Moreover, it will turn out that with one exception, for each $d \in \mathrm{N}$ there is at most one distinguished orbit of codimension $d$ in $\mathrm{g}$ ( $G$ exceptional). If we are not in this special case we can then clearly find the induced orbit.

The exception occurs with $E_{8}$ when $p=2$ and $d=22$. We have then two distinguished orbits. As they will both be obtained by induction, the corresponding representations of $W$ in Springer's parametrization can be determined [10], and this allows to compute induction in the remaining cases where $\operatorname{dim} M_{x}=22$.

Remark. The method described above works also for unipotent classes of exceptional groups, with the advantage that the difficulty with $E_{8}, p=2, d=22$ doesn't occur. This method seems to be more systematic than those devised so far (see e.g. [15]). 


\section{Construction of distinguished orbits}

For $E_{7}$ and $E_{8}$ in bad characteristic we don't know yet that the number of nilpotent orbits is finite.

Let $C_{1}, \ldots, C_{m}$ be the non-distinguished nilpotent orbits in $\mathrm{g}$. The number $\sum_{1 \leqslant i \leqslant m}\left|C_{i}^{F}\right|$ can be viewed as a polynomial in $q$. The results obtained so far allow to compute it for $E_{7}$, and also for $E_{8}$ once we have representatives for the distinguished nilpotent orbits in the case of $E_{7}$. If the number of nilpotent orbits is finite and $C_{m+1}, \ldots, C_{n}$ are the distinguished ones, let $x_{i} \in C_{i}$ and $d_{i}=\operatorname{dim} G_{x_{i}}$ $(m<i \leqslant n)$. Thanks to a result of Springer [19], we have

$$
\sum_{m<i \leqslant n} q^{-d_{i}}=\left|G^{F}\right|^{-1}\left(q^{2 N}-\sum_{1 \leqslant i \leqslant m}\left|C_{i}^{F_{i}}\right|\right)
$$

where $N=\left|\Phi^{+}\right|$. This gives $n-m$ and $d_{m+1}, \ldots, d_{n}$ (up to permutation).

Let $b_{i}=\frac{1}{2}\left(d_{i}-\operatorname{dim} T\right)$. We shall obtain the distinguished orbits by the process of induction, and this will ensure that $\operatorname{dim} G_{x_{i}}=2 \operatorname{dim} \mathfrak{B}_{x_{i}}^{G}+\operatorname{dim} T$ $(m<i \leqslant n)$, so that $b_{i}=\operatorname{dim} \mathfrak{B}_{x_{i}}^{G}$.

If the characteristic is 0 and $C$ is a distinguished orbit, there exist a parabolic subgroup $P$ of $G$ such that $C$ contains a dense open subset of $\mathfrak{u}_{P}$ (that is $C$ is a Richardson orbit). Moreover there is a canonical choice for $P$, up to conjugation [2]. In bad characteristic we can take the corresponding class of parabolic subgroups and the associated Richardson orbit. This orbit, the existence of which is not obvious at this stage, turns out to be distinguished. We call such orbits standard distinguished orbits. The corresponding values of $b_{i}$ are all distinct (for exceptional groups). For $E_{7}$ and $E_{8}$ they are

$$
\begin{aligned}
& E_{7}: 0,1,2,3,5,7 ; \\
& E_{8}: 0,1,2,3,4,5,6,7,8,10,16 .
\end{aligned}
$$

In bad characteristic we expect the following additional values:

$$
\begin{aligned}
& E_{7}, p=2: 6 \\
& E_{7}, p=3: \text { none } \\
& E_{8}, p=2: 7,9,13 \text { (notice that } 7 \text { is repeated); } \\
& E_{8}, p=3: 11 ; \\
& E_{8}, p=5: \text { none. }
\end{aligned}
$$

Comparing with characteristic 0 , we find that for some non-distinguished nilpotent orbits $\operatorname{dim} G_{x}$ is larger than expected. For example, if $G$ is of type $E_{7}$ and $p=2$, the orbits $A_{3}+A_{2}$ and $A_{6}$ give stabilizers of dimension 37 and 21 respectively, instead of 35,19 . For $A_{3}+A_{2}$ the difference (in the number of 
$F$-stable nilpotent elements) is made up by an extra distinguished orbit in $D_{6}$, and the extra distinguished orbit in $g$ (with $b_{i}=6$ ) should make up for $A_{6}$. In characteristic 0 the orbit $A_{6}$ is the Richardson orbit corresponding to the parabolic subgroups with Levi factors of type $A_{2}+3 A_{1}$. In characteristic 2 the extra distinguished orbit should be the corresponding Richardson orbit in $g$.

In a similar way we find that for $E_{8}, p=2$, the extra distinguished orbits in $g$ should take the place of $D_{7}\left(b_{i}=7\right), D_{7}\left(a_{1}\right)\left(b_{i}=9\right)$ and $D_{5}+A_{2}\left(b_{i}=13\right)$. For $E_{8}, p=3$, the extra distinguished orbit should take the place of $A_{7}$.

As in paragraph 3 we shall use the computer to calculate $\left|U_{z}^{F}\right|$ for various elements $z \in u^{F}$, in view to find $\operatorname{dim} U_{z}$. As $U_{z}$ can now be disconnected, we get only the following

Test. Let $d \in \mathbf{N}$. If $\left|U_{z}^{F}\right|<q^{d+1}$, then $\operatorname{dim} U_{z} \leqslant d$.

On the other hand it is clear that if $\operatorname{dim} U_{z}=d$, then $\left|U_{z}^{F}\right|<q^{d+1}$ if $q$ is large enough. In this paper it will be enough to take $q \leqslant 5$ to get the required results.

We describe now how to get distinguished orbits.

Choose a parabolic subgroup $P$ of $G$, a Levi factor $M$ of $P$ and a nilpotent orbit $C$ in $m$ in such a way that in characteristic 0 the induced orbit would be the expected one. We can take $P$ maximal, $P \supset B, M \supset T$, and we choose $x \in C^{F}$ such that $B$ and $T$ contain respectively a Borel subgroup and a maximal torus of $M_{x}$.

Let $H=M_{x} U_{p}$. We find a suitable subset $E \subset\left\{\lambda \in \Phi \mid X_{\lambda} \in \mathcal{u}_{P}\right\}$ such that $V=\sum_{\lambda \in E} k X_{\lambda}$ is $H$-stable (see however the remark at the end of the paragraph). Then $H$ acts on $Y=\left(x+\mathfrak{u}_{P}\right) / V$. This action should be easy to work with. For $y \in x+u_{P}$, let $\bar{y}=y+V \in Y$. Then $P_{y} \subset H_{\bar{y}}$. We are interested in the following special cases.

Case 1. We can find $y \in x+\mathfrak{u}_{P}^{F}$ such that $H_{\bar{y}}^{0} \subset U$ and $\operatorname{dim} U_{y} \leqslant \operatorname{dim} M_{x}$. Then the induced orbit $\tilde{C}$ exists, it is distinguished, $y \in \tilde{C}$ and $G_{y}^{0} \subset U$.

Case 2. We can find $y \in x+u_{P}^{F}$ such that

(i) the orbit of $\bar{y}$ is dense in $Y$.

(ii) Let $K=H_{\bar{y}}$. Then $K^{0} / R_{u}\left(K^{0}\right)$ is either a torus or is of type $A_{1}$.

(iii) $B, T$ contain respectively a Borel subgroup and a maximal torus of $K$.

Using the method described in paragraph 5 , we check first that the induced orbit, if it exists, is distinguished. This shows that there exists a dense open subset of $y+V$ consisting of distinguished elements. Let $d=\operatorname{dim} M_{x}$.

(a) If $K^{0} . / R_{u}\left(K^{0}\right)$ is a torus, it is enough to find $z \in y+V^{F}$ such that $\operatorname{dim} U_{z} \leqslant d$. Indeed, by semicontinuity the set $V_{0}=\left\{v \in V \mid \operatorname{dim} U_{y+v} \leqslant d\right\}$ is 
then open dense in $V$. There exists therefore a distinguished element $\tilde{x} \in y+V_{0}$. Then $K_{\tilde{x}}^{0} \subset G_{\tilde{x}}^{0} \cap B \subset U$. Therefore $\operatorname{dim} K_{\tilde{x}}=\operatorname{dim} U_{\tilde{x}} \leqslant d$, as required.

(b) If $K^{0} / R_{u}\left(K^{0}\right)$ is of type $A_{1}$, we show first that there exists $z \in y+V^{F}$ such that $\operatorname{dim} U_{z} \leqslant d-1$. Then $V_{0}=\left\{v \in V \mid \operatorname{dim} U_{y+v} \leqslant d-1\right\}$ is open dense in $V$. If $\tilde{x} \in y+V_{0}$ is distinguished, then $K_{\tilde{x}}^{0}$ is unipotent, and therefore $\operatorname{dim} K_{\tilde{x}} \leqslant$ $\operatorname{dim} U_{\tilde{x}}+1 \leqslant d$, as required.

(c) If $K^{0} / R_{u}\left(K^{0}\right)$ is simple of type $A_{1}$, we proceed as in (b). Let $e=\operatorname{dim} V$ and let $C_{0}=K \cdot \tilde{x}$. Then $\left|C_{0}^{F}\right|=\left(q^{2}-1\right) q^{e-2}$. For $z^{\prime} \in y+V, z^{\prime} \notin C_{0}$, we must therefore have $\operatorname{dim} K_{z^{\prime}} \geqslant d+2$, and also $\operatorname{dim} U_{z^{\prime}} \geqslant d$. This shows that $z \in G \cdot \tilde{x}$.

Notice that in cases 1 and 2(c) we get an explicit representative for the distinguished orbit, but not in cases 2(a) and 2(b).

It will be convenient to write $\bar{E}=\left\{\lambda \in \Phi \mid X_{\lambda} \in \mathfrak{u}_{P}\right.$ and $\left.\lambda \notin E\right\}$.

ExAmple. If $C$ is a distinguished orbit in $\mathfrak{m}$, let $\alpha$ be the unique element of $\Delta$ such that $X_{\alpha} \in \mathfrak{u}_{p}$. We take $\bar{E}=\{\alpha\}$. We are in case 1, with $y=x+X_{\alpha}$. The corresponding situation for unipotent elements is discussed in [10].

This gives already standard distinguished orbits for the following values of $b_{i}$ : $E_{7}: 0,1,2,3$;

$E_{8}: 0,1,2,3,5,7$ (assuming that the case of $E_{7}$ has been successfully dealt with).

REMARK. If $E^{\prime} \supset E$ and $V^{\prime}=\sum_{\lambda \in E^{\prime}} k X_{\lambda}$ have properties similar to those of $E$ and $V$, we can choose $y^{\prime} \in x+\mathfrak{u}_{P}^{F}$ in the same way as $y$ is chosen in case 2 , and then restrict our attention to the action of $K^{\prime}=H_{\bar{y}^{\prime}}$ on $y^{\prime}+V^{\prime}$ and $\left(y^{\prime}+V^{\prime}\right) / V$, where $\bar{y}^{\prime}=y^{\prime}+V^{\prime}$. In particular it is sufficient now for $V$ to be $K^{\prime}$-stable. We write also $\bar{E}^{\prime}=\left\{\lambda \in \Phi \mid X_{\lambda} \in \mathfrak{u}_{P}\right.$ and $\left.\lambda \notin E^{\prime}\right\}$.

\section{Standard distinguished orbits}

Let $G$ be a group of type $E_{6}$ and consider the following elements of $\mathrm{g}$ :

$$
\begin{aligned}
x^{(1)}= & X_{10000}+X_{00001}+X_{11000} \\
& +X_{00011}+X_{00100}+X_{01110} \\
x^{(2)}= & X_{11000}+X_{01110}+X_{01100} \\
& +X_{00110}+X_{00111} \\
x^{(3)}= & X_{11000}+X_{00011}+X_{00111}+X_{01210} \\
x^{(4)}= & X_{11110}+X_{01111}+X_{11100} \\
& +X_{00111}+X_{01210}
\end{aligned}
$$


Their orbits are respectively $E_{6}\left(a_{3}\right), A_{4}+A_{1}, D_{4}\left(a_{1}\right)$ and $2 A_{2}+A_{1}$. Let $x=x^{(i)}(1 \leqslant i \leqslant 4)$. Then $B, T$ contain respectively a Borel subgroup and a maximal torus of $G_{x}$. Let $u=\Pi_{\lambda>0} x_{\lambda}\left(c_{\lambda}\right)$. Suppose that $u \in G_{x}$. Then:

(a) if $i=2$,

$$
c_{00001}=0 \Rightarrow \underset{0}{c_{00011}}=0 .
$$

(b) if $i=3$,

$$
c_{00001}=c_{00100}=c_{00110}=c_{00111}=0 .
$$

Moreover, if $i=1$, then $G_{x}^{0} \subset U$. If $i=4$, then $G_{x}^{0} / R_{u}\left(G_{x}^{0}\right)$ is simple of type $A_{1}$, and

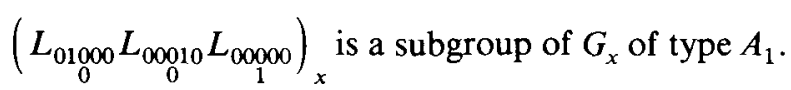

These statements follow easily from results in [17] and the commutation formulae.

If now $G$ is of type $E_{7}$ or $E_{8}$, we consider the elements above as elements of $g$ in the obvious manner.

We have already constructed some standard distinguished orbits in the previous paragraph. The remaining ones correspond to $b_{i}=5,7$ for $E_{7}$, and $b_{i}=4,6,8$, 10,16 for $E_{8}$. The characteristic is assumed to be bad.

(A) For $E_{7}$ we must find representatives to be able to carry on with $E_{8}$. In both cases we take $M$ of type $E_{6}$ and

$$
\bar{E}^{\prime}=\left\{\begin{array}{c}
000001 \\
0
\end{array}\right\}, \quad y^{\prime}=x+X_{000001}
$$

(the notation is the same as in paragraph 6).

$$
b_{i}=5 \text {. Let } x=x^{(2)} \text {, }
$$

$$
\bar{E}=\bar{E}^{\prime} \cup\left\{\begin{array}{cc}
000011 & 000111 \\
0 & 0
\end{array}\right\}
$$

Taking

$$
y=y^{\prime}+X_{000111}
$$

we are in case 1 of paragraph 6 , as can be seen in particular from the remarks at the beginning of this paragraph. The inequality $\operatorname{dim} U_{y} \leqslant \operatorname{dim} M_{x}$ is obtained with $q=p$. Thus the induced class $\tilde{C}$ exists, $y \in \tilde{C}$ and $G_{y}^{0} \subset U$.

$b_{i}=7$. Let $x=x^{(3)}$,

$$
\bar{E}=\bar{E}^{\prime} \cup\left\{\begin{array}{ccc}
000011 & 000111 & 001111 \\
0 & 0 & 0
\end{array}\right\} .
$$

Taking

$$
y=y^{\prime}+X_{000011}+\underset{0}{00111}
$$

we are in case 1 . We can use $q=p$. Thus $\tilde{C}$ exists, $y \in \tilde{C}$ and $G_{y}^{0} \subset U$. 
It follows that $B$ contains a Borel subgroup of $M_{x}$ and $M_{x}^{0} / R_{u}\left(M_{x}^{0}\right)$ is of type $3 A_{1}$. An easy computation with the commutation formulae shows that if $u=$ $\Pi_{\lambda>0} x_{\lambda}\left(c_{\lambda}\right) \in M_{x}$, then

$$
c_{0010000}=c_{0000000}=c_{0010000}=0 .
$$

It follows that we can take

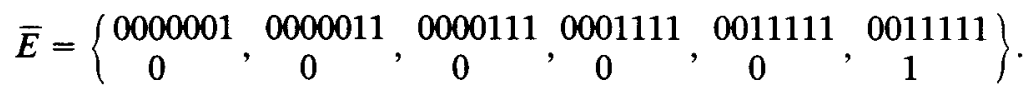

We are then in case $2(a)$. We use

$$
\begin{gathered}
y=x+X_{0001111}+X_{0011111}+X_{0011111}, \\
z=y, \quad q=p .
\end{gathered}
$$

\section{Non-standard distinguished orbits}

For $E_{7}$ it remains to construct one non-standard distinguished orbit if $p=2$; for $E_{8}$, three non-standard distinguished orbits if $p=2$, and one if $p=3$.

In each case the proof that $B, T$ contain respectively a Borel subgroup and a maximal torus of $M_{x}$ is omitted. One can use arguments similar to those invoked in the case of the standard distinguished orbit in $E_{8}$ for which $b_{i}=16$.

(A) For $E_{7}$, we take $M$ of type $D_{5}+A_{1}$, with

$$
x=X_{111000}+X_{011100}+X_{011000}+X_{001100},
$$

in the orbit $\left(A_{2}+2 A_{1} ; \varnothing\right)$. Let $L$ be the subgroup of $M$ generated by

$$
L_{100000}, \underset{0}{L_{010000}}, \underset{0}{L_{000100}} \text { and } L_{000000} \text {. }
$$

Then $L_{x}$ is simple of type $A_{1}$. We also have

$$
L_{000001} \subset M_{x},
$$

and $M_{x}^{0} / R_{u}\left(M_{x}^{0}\right)$ is of type $2 A_{1}$. Let

$$
\bar{E}=\left\{\begin{array}{cccc}
000010 & 000110 & 000011 & 000111 \\
0 & 0 & 0 & 0
\end{array}\right\} .
$$

We are then in case 2 (c). We can use

$$
\begin{aligned}
& y=x+X_{000110}+X_{000011}, \\
& z=y+X_{001111}, \quad q=2 .
\end{aligned}
$$

Thus $\tilde{C}$ exists and $z \in \tilde{C}$. 
(B) Suppose now that $G$ is of type $E_{8}$ and $p=2$.

$b_{i}=7$. Let $M$ be of type $D_{7}$ and let

$$
x=X_{0110000}+X_{0011100}+X_{0001110}+X_{0} X_{0} X_{11000}+X_{0011000}+X_{0000111} \text {, }
$$

in the orbit $2 A_{3}$. Then

$$
\left(L_{0}^{L_{0010000}} L_{0000010}\right)_{x}
$$

is of type $A_{1}$, and $M_{x}^{0} / R_{u}\left(M_{x}^{0}\right)$ is of type $A_{1}$. We can take

$$
\bar{E}=\left\{\begin{array}{c}
1000000 \\
0
\end{array}\right\} \text {. }
$$

We are in case 2(c). We can use

$$
\begin{gathered}
y=x+X_{1000000}, \\
z=y+X_{1122100}, \quad q=2 .
\end{gathered}
$$

Thus $\tilde{C}$ exists and $z \in \tilde{C}$.

$b_{i}=9$. Let $M$ be of type $D_{5}+A_{2}$ and let

$$
x=X_{1111000}+X_{1110000}+X_{0121000}+X_{0000011},
$$

in the orbit $\left(3 A_{1} ; A_{1}\right)$. Then

$$
\left(\operatorname{Lop0000}_{0}^{L_{0000000}}\right)_{x}
$$

is of type $A_{1}$,

$$
\underset{0}{L_{0100000}} \subset M_{x}
$$

and $M_{x}^{0} / R_{u}\left(M_{x}^{0}\right)$ is of type $2 A_{1}$. We can take

$$
\bar{E}=\left\{\begin{array}{cc}
0000100 & 0001100 \\
0 & 0
\end{array}\right\} .
$$

We are in case $2(b)$. We use

$$
\begin{gathered}
y=x+X_{0001100}, \\
z=y+\underset{0}{X_{0000110}}+\underset{0}{X_{0011111}}+X_{1232100}, \\
q=2 .
\end{gathered}
$$

$b_{i}=13$. Let $M$ be of type $E_{7}$ and let

$$
\begin{aligned}
x= & X_{1111100}+X_{0111110}+X_{1111000} \\
& +X_{0111100}+X_{0011110}+X_{0121000}
\end{aligned}
$$


in the orbit $A_{3}+A_{2}+A_{1}$. Let $L^{\prime} \supset T$ be a Levi factor of the parabolic subgroup of $G$ generated by $B$ and

$$
L_{1110000}
$$

A direct computation shows that

$$
\left(L^{\prime} L_{0000110}\right)_{x}
$$

is of type $A_{1}$, and $M_{x}^{0} / R_{u}\left(M_{x}^{0}\right)$ is also of type $A_{1}$. We can take

$$
\bar{E}=\left\{\begin{array}{ccc}
0000001 & 0000011 & 0000111 \\
0 & 0 & 0
\end{array}\right\}
$$

We are then in case 1 with

$$
y=x+X_{0000011}+X_{0000111} .
$$

The required inequality is obtained with $q=2$.

(C) Let now $G$ be of type $E_{8}$ with $p=3$. We must find a distinguished orbit with $b_{i}=11$. We take $M$ of type $D_{5}+A_{2}$ and

$$
x=X_{1111000}+X_{1110000}+X_{0121000} \text {, }
$$

in the orbit $\left(3 A_{1} ; \varnothing\right)$. Then $M_{x}^{0} / R_{u}\left(M_{x}^{0}\right)$ is of type $A_{2}+2 A_{1}$ and the factors $A_{1}$ are given by

$$
\underset{0}{L_{010000}}
$$

and

$$
\left(L_{1000000} L_{0001000}\right)_{x}
$$

We take first

$$
\bar{E}^{\prime}=\left\{\begin{array}{cccccc}
0000100 & 0001100 & 0000110 & 000111 & 0000111 & 0001111 \\
0 & 0 & 0 & 0 & 0 & 0
\end{array}\right\},
$$

with

$$
y^{\prime}=x+\underset{0}{X_{000110}}+X_{0000111}
$$

We can take

$$
\bar{E}=\bar{E}^{\prime} \cup\left\{\begin{array}{ccc}
0011100 & 0111100 & 0011100 \\
0 & 0 & 1
\end{array}\right\}
$$

and we are in case 2(c). We use

$$
y=y^{\prime}+X_{0111100}+X_{0011100}, \quad z=y+X_{0011111}, \quad q=3 .
$$

Thus $\tilde{C}$ exists and $z \in \tilde{C}$.

REMARK. In the case of $E_{8}, p=2$, it remains to show that the two distinguished orbits $C, C^{\prime}$ we have constructed for $b_{i}=7$ are distinct. 
Let $M$ be of type $E_{7}$ and let $C_{1} \subset \mathrm{m}$ be the distinguished $M$-orbit for which $b_{i}=7$. In paragraph 6 we have constructed a distinguished orbit $C$ in $g$ by inducing $C_{1}$ from $\mathrm{m}$ to $\mathrm{g}$. Since $G / P$ is complete, $\bar{C}=G \cdot\left(\bar{C}_{1}+\mathfrak{u}_{P}\right)$. The elements in $C_{1}+\mathfrak{u}_{P}$ are all contained in $C$ or in the closure of the distinguished orbit in $\mathrm{g}$ for which $b_{i}=8$ (this follows from results in [10] and the way this orbit is obtained in paragraph 7). If the orbit $D_{7}$ is contained in $\bar{C}$, it must then be induced from an orbit $C_{2} \subset \bar{C}_{1}$ with $C_{2}$ of codimension 2 in $\bar{C}_{1}$. The only possibilities for $C_{2}$ are $D_{6}\left(a_{2}\right)$ and $E_{6}\left(a_{3}\right)$, and they cannot give $D_{7}$ by induction.

The orbit $D_{7}$ of $G$ is therefore not contained in $\bar{C}$. But it is obviously in the closure of the distinguished orbit $C^{\prime}$ constructed in this paragraph. Thus $C \neq C^{\prime}$.

\section{The classification of nilpotent orbits}

In paragraph 6 we have defined standard distinguished orbits. More generally, for $x \in \mathscr{N}$, let $S$ be a maximal torus of $G_{x}$ and let $M=C_{G}(S)$. We say that the orbit of $x$ in $\mathfrak{g}$ is standard if $M \cdot x$ is a standard distinguished orbit in $g$. Let $d_{x}=\operatorname{dim} G_{x}$. If $x$ is standard, we can also define $d_{x}^{0}$ to be the dimension of the centralizer of a corresponding element in characteristic 0 .

We have proved

TheOREM 3. Let $G$ be of type $E_{7}$ or $E_{8}$ and let $x \in \mathcal{N}$. As above let $S$ be a maximal torus of $G_{x}$ and let $M=C_{G}(S)$. Then:

(a) If $G \cdot x$ is standard, then $d_{x}=d_{x}^{0}$, except for the orbit $D_{4}+A_{2}$ if $G$ is of type $E_{8}$ and $p=2$, in which case $d_{x}=d_{x}^{0}+6$, and in the following cases where

$$
\begin{aligned}
& d_{x}=d_{x}^{0}+2: \\
& E_{7}, p=2: A_{3}+A_{2}, A_{6} ; \\
& E_{8}, p=2: A_{3}+A_{2}, A_{6}, D_{5}+A_{2}, D_{7}, D_{7}\left(a_{1}\right) ; \\
& E_{8}, p=3: A_{7} .
\end{aligned}
$$

(b) If $G \cdot x$ is non-standard, we are in one of the following cases.

(i) $p=2, M$ is of type $D_{6}, d_{x}=37$ (resp. 72) if $G$ is of type $E_{7}$ (resp. $E_{8}$ ) and $M \cdot x$ is the only non-standard distinguished orbit in $\mathrm{m}$.

(ii) $p=2, M$ is of type $E_{7}, d_{x}=19$ (resp. 40 ) if $G$ is of type $E_{7}$ (resp. $E_{8}$ ) and $M \cdot x$ is the only non-standard distinguished orbit in $\mathrm{m}$.

(iii) $p=2, M$ is of type $D_{7}, d_{x}=56$ and $M \cdot x$ is the only non-standard distinguished orbit in $\mathrm{m}$.

(iv) $p=2, M=G$ is of type $E_{8}$ and $G \cdot x$ is one of the three non-standard distinguished orbits in $\mathrm{g}$. Moreover the possible values for $d_{x}$ are 22, 26, 34 . 
(v) $p=3, M=G$ is of type $E_{8}, d_{x}=30$ and $G \cdot x$ is the only non-standard distinguished orbit in $\mathfrak{g}$.

REMARK. In [16] a different approach is used, and the orbits listed in part (a) of the theorem appear as the 'new' orbits, and those in part (b) as corresponding to orbits existing in characteristic 0 .

We have proved also

TheOREM 4. Let $G$ be of type $F_{4}$, with $p=3$. Then all nilpotent orbits in $g$ are standard, and $d_{x}=d_{x}^{0}$ for all $x \in \mathscr{N}$.

\section{Appendix}

The Computation of Centralizers in Finite $p$-groups

The computational problem encountered above was the following. We are given a finite $p$-group $G$ of order $q^{n}$, where $q$ is a power of $p$, which acts in a prescribed manner on an elementary abelian $p$-group $N$, also of order $q^{n}$, and we want to calculate the order of the centralizer in $G$ of various elements of $N$. In fact $G$ is a unipotent subgroup of one of the finite Chevalley groups $E_{7}(q)$ and $E_{8}(q)$, in which case $n$ is 63 or 120 respectively, and $N$ is the underlying vector space of the corresponding Lie Algebra. The relevant values of $q$ are 2, 4, 3 and 5. Equivalently, we are asking for the centralizer of an element of $N$ in the semidirect product $G N$, and so we can consider this problem as a special case of the problem of computing centralizers of elements in finite $p$-groups. An efficient algorithm for doing this and for computing conjugacy classes in p-groups is described by Felsch and Neubüser in [6]. In fact, we used a somewhat simpler method, which would probably be slower in general, but was adequate for these particular examples. We shall now give a brief description of this method.

It is convenient to define our $p$-group $P$ by means of a power-commutator presentation. This means that we have generators $x_{1}, x_{2}, \ldots, x_{n}$ of $P$, where $|P|=p^{n}$, such that

$$
1 \subset\left\langle x_{1}\right\rangle \subset\left\langle x_{1}, x_{2}\right\rangle \subset \cdots \subset\left\langle x_{1}, x_{2}, \ldots, x_{n}\right\rangle=P
$$

is a central series for $P$, and we are given the values of the commutators $\left[x_{i}, x_{j}\right]$ $(i<j)$ and the powers $x_{i}^{P}$. Then every element $x \in P$ has a unique expression of 
the form

$$
x=x_{n}^{i_{n}} \cdots x_{2}^{i_{2}} x_{1}^{i_{1}} \quad\left(0 \leqslant i_{j}<p\right)
$$

which we call the normal form of $x$. We assume that the commutators and powers are given in their normal form. Then these commutators and powers can be used in the so-called collection process (see, for example, [24]) to put an arbitrary word in the $x_{i}$ into normal form. This collection process can be carried out very efficiently on a machine, and it forms the basis of most computer algorithms for dealing with nilpotent groups. In the normal form for $x$ given above, we will call $x_{j}^{i_{j}}$ the leading term of $x$, where $j$ is maximal such that $i_{j} \neq 0$. Note that the leading term of an element is not changed when we replace the element by a conjugate.

In our specific example, $P=G N$, the generators of $G$ and $N$ are in one-one correspondence with the positive roots of the Lie Algebra $E_{7}$ or $E_{8}$. If $g$ and $h$ are generators of $G$ corresponding to roots $\phi$ and $\psi$ respectively, then $[g, h] \neq 1$ if and only if $\phi+\psi$ is also a root, in which case $[g, h]=k^{ \pm 1}$, where $k$ corresponds to the root $\phi+\psi$. The same rule applies to the action of $G$ on $N$. Furthermore, the $p^{\text {th }}$ powers of all of the generators are trivial. This, together with the fact that all commutators have length 0 or 1 , renders the collection process particularly easy in this case. (Of course, in the case $q=4$, the number of power-commutator generators of $G$ is really twice the number of roots, and commutators may have length 2 in these generators.) The chief difficulty pertaining to the input of this data was the sign in the expression $[g, h]=k^{ \pm 1}$, which is only a problem when $p$ is odd. The essential condition to be fulfilled here is that the relations in the presentation should be consistent, which means in effect that the associative law $\left(x_{i} x_{j}\right) x_{k}=x_{i}\left(x_{j} x_{k}\right)$ should be valid for all $i, j$ and $k$. Values for these signs were originally taken from Table 12, at the end of Mizuno's paper [12]. The computer was then used to check consistency, and about six errors were found and corrected.

Now suppose that we wish to find generators of the centralizer of an element $t$ in $P$. We assume that, at the $i$ th step of the computation $(1 \leqslant i \leqslant n)$, we have already found generators of the centralizer of $t$ in $\left\langle x_{1}, \ldots, x_{i-1}\right\rangle$, and we want to find out whether or not there exists $x_{i} w \in C(t)$, for some $w \in\left\langle x_{1}, \ldots, x_{i-1}\right\rangle$. If so, then $\left[x_{i}, t\right]$ and $\left[w^{-1}, t\right]$ will have the same leading term, and so we will keep a record of the leading terms that can occur as commutators with $t$, at each stage. At the $i$ th stage, we either find an element $x_{i} w \in C(t)$, or we find a commutator $\left[x_{i} w, t\right]$ for which the leading term generator has not occurred previously, and we record this commutator. We shall now write down this algorithm more precisely. $C$ will be the set of generators of $C(t)$, and, for $1 \leqslant i \leqslant n, b[i]$ will be an element of $P$ with $[b[i], t]=c[i]$, where $c[i]$ has some power of $x_{i}$ as its leading term. 


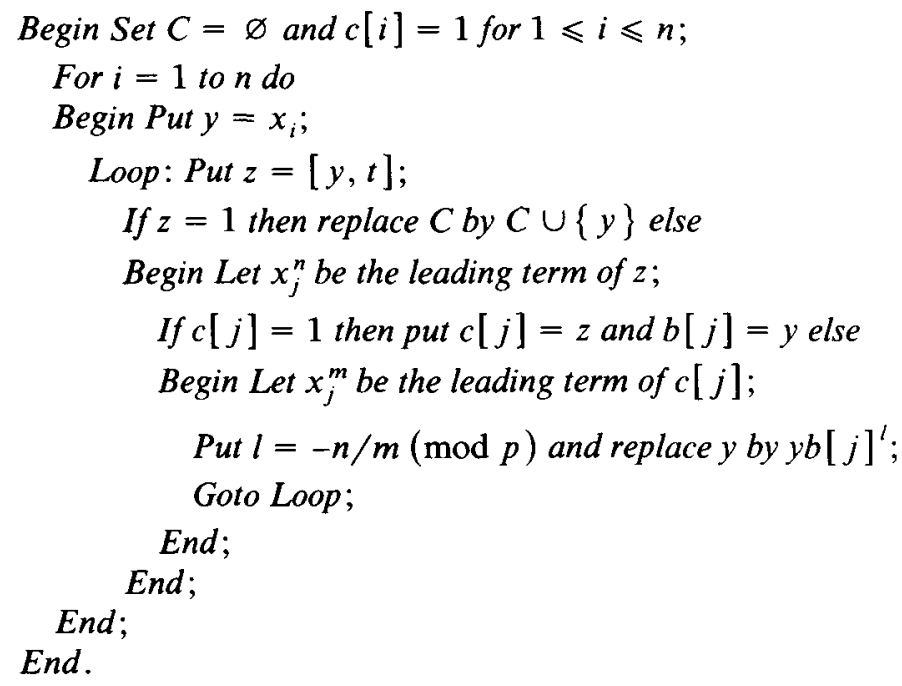

Using an implementation of this algorithm written in Burroughs Algol on the B6700 machine at Warwick University, a typical process time for the computation of the centralizer of an element in the case $E_{8}$, with $q=3$ or 4 was 10 seconds. It was rather longer than this in a few bad cases in which the elements $b[j]$ and $c[j]$ grew unusually long, but, owing to the simple nature of the power-commutator presentation, this did not happen very often. The Felsch-Neubüser method is completely different, and works downwards through the successive factor groups in the central series for $P$, rather than upwards through the subgroups, as we are doing here. We suspect that the downwards method would be ultimately more efficient if more complex presentations were involved.

\section{References}

[1] D. Alvis and G. Lusztig, 'On Springer's correspondence for simple groups of type $E_{n}$ $(n=6,7,8)$ ', Math. Proc. Cambridge Phil. Soc. 92 (1982), 65-78.

[2] P. Bala and R. W. Carter, 'Classes of unipotent elements in simple algebraic groups', Math. Proc. Cambridge Philos. Soc. 79 (1976), 401-425; 80 (1976), 1-18.

[3] W. Borho and R. MacPherson, 'Représentations des groupes de Weyl et homologie d'intersection pour les variètés nilpotentes', C. R. Acad. Sci. Paris Sér. I Math. 292 (1981), n 15, 707-710.

[4] N. Bourbaki, Groupes et algèbres de Lie, chap. IV, V, VI (Paris, Hermann, 1968)

[5] W. H. Hesselink, 'Nilpotency in classical groups over a field of characteristic 2', Math. Z. 166 (1979), 165-181.

[6] V. Felsch and J. Neubüser, 'An algorithm for the computation of conjugacy classes and centralizers in p-groups', Symbolic and algebraic computation, Edited by $\mathrm{E} . \mathrm{W} . \mathrm{K}$. $\mathrm{Ng}$ (Lecture Notes in Computer Science 72, Berlin-Heidelberg-New York, Springer-Verlag, 1979), pp. 452-456. 
[7] R. H. Jeurissen, The automorphism groups of octave algebras (Doctoral dissertation, University of Utrecht).

[8] G. Lusztig, 'On the finiteness of the number of unipotent classes', Invent. Math. 34 (1976), 201-213.

[9] G. Lusztig, 'Green polynomials and singularities of unipotent classes', Advances in Math. 42 (1981), 169-178.

[10] G. Lusztig and N. Spaltenstein, 'Induced unipotent classes', J. London Math. Soc. 19 (1979), 41-52.

[11] K. Mizuno, 'The conjugate classes of Chevalley groups of type $E_{6}$ ', J. Fac. Sci. Univ. Tokyo Sec. 1 A Math. 24 (1977), 525-563.

[12] K. Mizuno, 'The conjugate classes of unipotent elements of the Chevalley groups $E_{7}$ and $E_{8}$ ', Tokyo J. Math. 3 (1980), 391-461.

[13] T. Shoji, 'The conjugate classes of Chevalley groups of type $\left(F_{4}\right)$ over finite fields of characteristic $p \neq 2$ ', J. Fac. Sci. Univ, Tokyo Sec. 1A Math. 21 (1974), 1-17.

[14] N. Spaltenstein, 'On the fixed point set of a unipotent element on the variety of Borel subgroups', Topology 16 (1977), 203-204.

[15] N. Spaltenstein, Classes unipotentes et sous-groupes de Borel, Lecture Notes in Math. 946., Berlin-Heidelberg-New York, Springer-Verlag (1982).

[16] N. Spaltenstein, 'Nilpotent classes and sheets of Lie algebras in bad characteristic', Math. $Z$. 181 (1982), 31-48.

[17] N. Spaltenstein, 'On unipotent and nilpotent elements of groups of type $E_{6}$ ', J. London Math. Soc. 27 (1983), 413-420.

[18] N. Spaltenstein, 'Nilpotent classes in Lie algebras of type $F_{4}$ over fields of characteristic 2', J. Fac. Sci. Univ. Tokyo Sect. 1A Math. 30 (1984), 517-524.

[19] T. A. Springer, 'The Steinberg function of a finite Lie algebra', Invent. Math. 58 (1980), 211-216.

[20] T. A. Springer, and R. Steinberg, 'Conjugacy classes', Seminar on algebraic groups and related finite groups, A. Borel et al. (Lecture Notes in Math. 131, Berlin-Heidelberg-New York, Springer-Verlag, 1970).

[21] R. Steinberg, Conjugacy classes in algebraic groups (Lecture Notes in Math. 366, Berlin-Heidelberg-New York, Springer-Verlag, 1974).

[22] R. Steinberg, Endomorphisms of linear algebraic groups (Memoirs Amer. Math. Soc. 80 (1968)).

[23] U. Stuhler, 'Unipotente und nilpotente Klassen in einfachen Gruppen und Lie-Algebren vom typ $G_{2}$ ', Nederl. Akad. Wetensch. Proc. Ser. A 74 (1971), 365-378.

[24] G. Havas and T. Nicholson, 'Collection', SYMSAC, Proceedings of the ACM symposium on symbolic and algebraic computation, (Association for Computing Machinery, New York, 1976), pp. 9-14.

Mathematics Institute

University of Warwick

Coventry

England
Mathematisches Institut der Universität Bern

Sidlerstrasse 5 3012 Bern

Switzerland 